\title{
Function and Regulation of Protein Kinase D in Oxidative Stress: A Tale of Isoforms
}

\author{
Mathias Cobbaut $\mathbb{D}^{1,2}$ and Johan Van Lint $\mathbb{D D}^{1,2}$ \\ ${ }^{1}$ Department of Cellular and Molecular Medicine, Faculty of Medicine, KU Leuven, Leuven, Belgium \\ ${ }^{2}$ Leuven Cancer Institute (LKI), KU Leuven, Leuven, Belgium \\ Correspondence should be addressed to Johan Van Lint; johan.vanlint@kuleuven.be
}

Received 27 October 2017; Accepted 19 February 2018; Published 26 April 2018

Academic Editor: Sabrina Büttner

Copyright (c) 2018 Mathias Cobbaut and Johan Van Lint. This is an open access article distributed under the Creative Commons Attribution License, which permits unrestricted use, distribution, and reproduction in any medium, provided the original work is properly cited.

\begin{abstract}
Oxidative stress is a condition that arises when cells are faced with levels of reactive oxygen species (ROS) that destabilize the homeostatic redox balance. High levels of ROS can cause damage to macromolecules including DNA, lipids, and proteins, eventually resulting in cell death. Moderate levels of ROS however serve as signaling molecules that can drive and potentiate several cellular phenotypes. Increased levels of ROS are associated with a number of diseases including neurological disorders and cancer. In cancer, increased ROS levels can contribute to cancer cell survival and proliferation via the activation of several signaling pathways. One of the downstream effectors of increased ROS is the protein kinase D (PKD) family of kinases. In this review, we will discuss the regulation and function of this family of ROS-activated kinases and describe their unique isoformspecific features, in terms of both kinase regulation and signaling output.
\end{abstract}

\section{Oxidative Stress: Causes and Consequences}

Oxidative stress is a condition that develops when the cellular redox balance is disturbed by an excessive buildup of reactive oxygen species (ROS). ROS mainly occur as a byproduct of normal cellular metabolism, due to the leak of $1-3 \%$ of electrons utilized in the mitochondrial electron transport chain for the reduction of oxygen to water, resulting in the production of superoxide [1]. Besides this "collateral" production of ROS, they are also produced deliberately. ROS (mainly $\mathrm{H}_{2} \mathrm{O}_{2}$ ) are generated by oxidases in peroxisomes, for example, during $\beta$-oxidation of fatty acids and flavin oxidase activity [2]. Furthermore, ROS are also produced in the endoplasmic reticulum during oxidation of maturating proteins in the ER, which helps to stabilize them during folding [3]. Another source of ROS is the production of $\mathrm{H}_{2} \mathrm{O}_{2}$ by nicotinamide adenine dinucleotide (NADPH) oxidase complexes (NOX) in granulocytes and macrophages to kill pathogens [4]. NOX enzymes are also activated by growth factor signaling. Via the activation of kinases and by oxidizing the active-site cysteines of Tyr and lipid phosphatases, the NOX-generated ROS can potentiate the growth factor signaling output [5-8]. While ROS produced in these contexts serve a purpose, their levels should be tightly controlled, since excessive levels of ROS can cause damage to macromolecules (such as DNA, proteins, and lipids) and cause severe mitochondrial damage, causing it to leak cytochrome $c$ resulting in apoptosis [9-16]. To this end, cells have several antioxidant mechanisms in place to prevent the excessive buildup of ROS. These are both enzymatic (e.g., superoxide dismutases, thioredoxin reductases, and glutaredoxins) and nonenzymatic (e.g., ascorbic acid, $\alpha$-tocopherol, and glutathione) in nature [17-20]. A disturbed redox balance is associated with a variety of pathologies, including cardiovascular disease, fibrosis, neurological disease, and cancer [21-25]. Interestingly, several cancer cell lines have been shown to harbor increased levels of ROS in comparison to nontransformed cells $[26,27]$. This elevated ROS is thought to come from diverse sources: altered metabolism and mitochondrial functions, mutations in mtDNA, enhanced growth factor signaling, and activation of oncogenes such as mutant forms of Ras and c-Myc [3, 28-30]. For example, it was shown that 


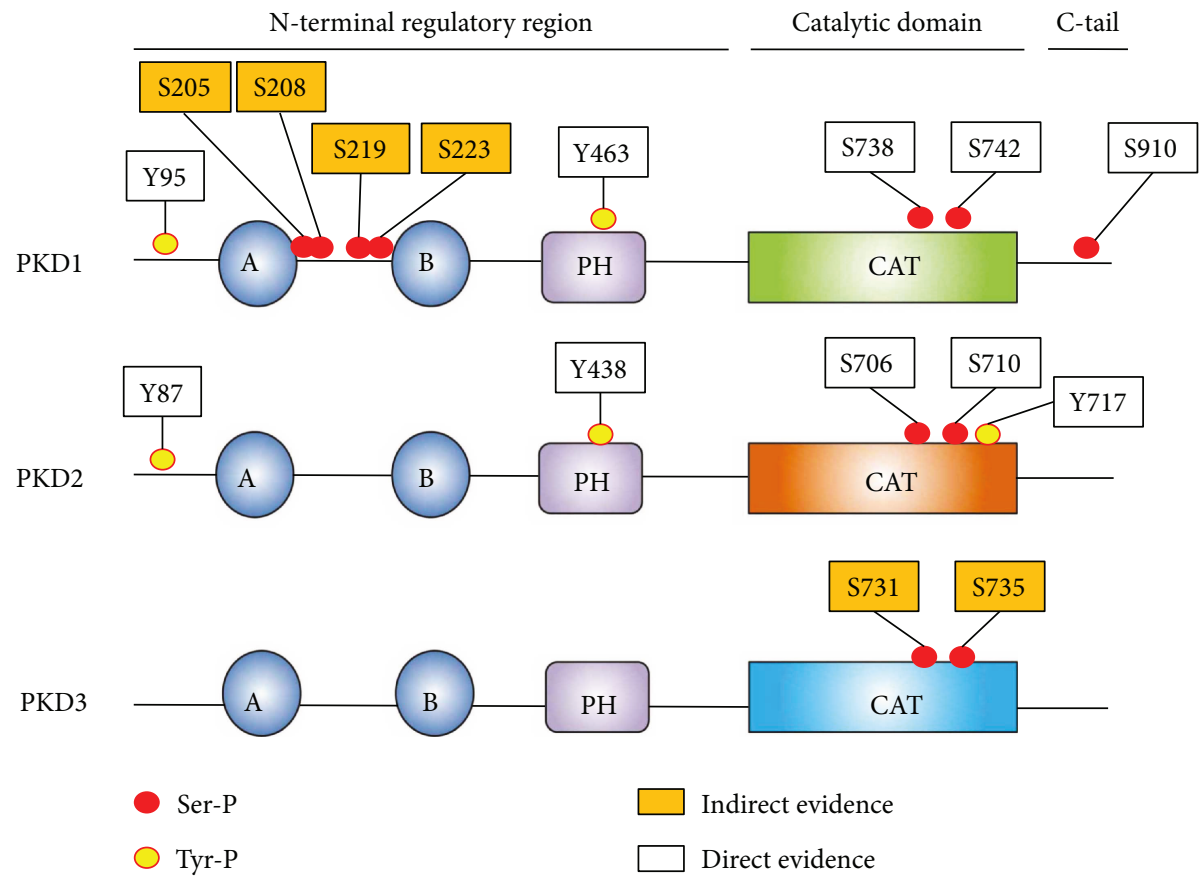

FIGURE 1: Domain organization and phosphorylation sites of PKD1/2/3 in oxidative stress conditions. Tyr phosphorylation sites are indicated with yellow-coloured circles, and Ser phosphorylation sites are indicated with red-coloured circles. "Direct evidence" indicates that the sites have been shown to be phosphorylated via immunoblotting with a site-specific antibody or via mass spectrometry. "Indirect evidence" indicates data obtained via site-directed mutagenesis or readouts dependent on general phosphorylation (such as PhosTag gels). A and B denote $\mathrm{Cla}$ and $\mathrm{C} 1 \mathrm{~b}$ domains, $\mathrm{PH}$ denotes the pleckstrin homology domain, and CAT denotes the kinase domain.

exogenous expression of $\mathrm{H}$-RasG12V in $3 \mathrm{~T} 3$ cells increases their proliferation rate, an event dependent on increased ROS levels [31]. Furthermore it was shown that $\mathrm{H}$ RasG12V increases ROS levels by activating NOX4 [32]. Besides Ras, c-Myc has also been shown to increase ROS levels in cancer cells, leading to DNA damage and genomic instability, thereby promoting cancer development [33, 34]. Moderate levels of ROS result in the activation of several signaling cascades contributing to either increased proliferation or enhanced survival of cancer cells. For example, upregulation of the PI3K/Akt pathway (e.g., via the inactivation of PTEN), MAP kinase pathways such as Erk1/2 and JNK (although the latter is also involved in ROS-induced apoptosis), and the NF- $\kappa \mathrm{B}$ pathway has been observed [31, 35-37]. An important downstream regulator of the oxidative stress response is protein kinase $\mathrm{D}(\mathrm{PKD})$. The mechanisms leading to the activation of different PKD isoforms in cells undergoing oxidative stress, as well as the signaling consequences of this activation will be discussed in this review.

\section{Protein Kinase D}

2.1. The PKD Family: Isoforms and Domain Organization. The human PKD family consists of three isoforms in humans (PKD1, PKD2, and PKD3) and belongs to the $\mathrm{Ca}^{2+} /$ calmodulin-dependent protein kinase (CAMK) group of Ser/Thr kinases. PKD1 is the largest member, with 912 amino acids and a molecular mass of $115 \mathrm{kDa}$. PKD2 and PKD3 are smaller with molecular masses of $105 \mathrm{kDa}$ and $110 \mathrm{kDa}$, respectively [38].
PKDs are modular enzymes that contain a long $\mathrm{N}$-terminal regulatory region followed by a catalytic domain and a C-terminal extension (C-tail) (Figure 1). The N-terminal part of the protein contains several regions and domains involved in kinase autoregulation, localization, and binding to interactors.

At the extreme N-terminus, PKD1/2 contain a hydrophobic Ala(/Pro)-rich stretch (not found in PKD3), which has been hypothesized to insert in membranes [39]. This region is followed by two diacylglycerol (DAG) binding $\mathrm{C} 1$ domains. Because of this feature, PKDs were first classified as members of the protein kinase C (PKC) family [40]. However, the catalytic domain of PKD1 shows higher homology CAM kinases and has similar substrate and inhibitor specificity which resulted in the classification of PKDs as members of the CAMK group [41, 42]. While the C1b domain binds PDB with high affinity, DAG is preferably bound by the $\mathrm{Cla}$ domain [43]. In PKD, the C1a and C1b domains are separated by a large linker of approximately 70 amino acids (compared to the linker in PKC isoforms that is much shorter, for example, 8 amino acids in cPKC isoforms). Additionally, the C1a-C1b linker in PKD has important functional properties. Phosphorylation of Ser-205/208 and Ser-219/223 in the linker has been shown to generate a 14-3-3 binding site, which is crucial for ASK1 binding and downstream JNK activation in $\mathrm{H}_{2} \mathrm{O}_{2}$-stimulated cells (see further [44]).

The C1 domains are connected to the pleckstrin homology $(\mathrm{PH})$ domain by a large linker enriched in acidic amino acids (sometimes denoted as the acidic domain). The 
potential regulatory role of this acidic stretch is not fully explored, but it has been suggested in an early study that it could play a role in PKD activation. This idea resulted from the observation that basic peptides and proteins (such as protamine sulfate, myelin basic protein, and histone $\mathrm{H} 1$ ) could inhibit $\mathrm{PKD}$ in vitro. On the other hand, polyanionic molecules such as heparin or dextran sulfate are capable of activating $\mathrm{PKD}$, without phosphatidylserine/12-O-tetradecanoylphorbol-13-acetate (PS/TPA) [45]. Therefore, the authors hypothesized that polyanionic molecules could disrupt an intramolecular interaction between the acidic domain and a basic stretch elsewhere in the protein [45]. This hypothesis however has not been further explored. The $\mathrm{PH}$ domain itself functions as a negative regulator of kinase activity. A full deletion, as well as partial deletions of the $\mathrm{PH}$ domain, renders the kinase constitutively active [46]. Pleckstrin homology domains are known interacting modules for phosphoinositides. However, only a small number of these have lipid-binding capabilities, for which the requirements are well defined [47-49]. Structural analysis of the available NMR structures of PKD PH domains in the protein databank (2COA and $2 \mathrm{D} 9 \mathrm{Z}$ ) reveals that the $\mathrm{PH}$ domains of PKD lack the necessary amino acids to interact with phosphatidylinositol phosphate head groups. Hence, the $\mathrm{PH}$ domain of PKD does not seem to serve as a lipidinteraction module, but rather serves as a protein interaction module. Important binding partners in the context of PKD activation are $\mathrm{G} \beta \gamma$ isoforms. Binding of PKD to $\mathrm{G} \beta \gamma$ heterodimers has been proven to directly activate immunoprecipitated PKD1 in vitro [50]. Also, incubation of permeabilized HeLa cells with G $\beta \gamma$ causes PKD activation, and when competing free $\mathrm{PH}$ domain was added, activation was decreased [50]. Seemingly in contrast to this finding, transfection studies showed that cotransfection of $\mathrm{G} \beta \gamma$ isoforms with phospholipase C (PLC) $\beta 2 / 3$ isoforms was necessary to activate PKD. However, only certain G $\beta \gamma$ isoforms could activate PKD 1 and PLC $\beta 2 / 3$, while other G $\beta \gamma$ isoforms could activate PLC $\beta 2 / 3$ but not PKD1 [51]. This indicates that structural compatibility between $\mathrm{G} \beta \gamma$ and the $\mathrm{PH}$ domain is required for activation of PKD, besides DAG generation by PLC $\beta 2 / 3$.

The N-terminal regulatory region is followed by the catalytic domain. Notably, PKDs are non-RD kinases, that is, they do not contain an Arg in their catalytic loop (HRD motif). However, while these non-RD kinases normally are not dependent on activation loop phosphorylation, PKDs are (in most cases) dependent on activation loop Ser-738/ 742 (hPKD1 numbering) phosphorylation for their activity [52]. The catalytic domain is followed by a C-terminal extension (C-tail). The C-terminal portion of the tail is not conserved and may contribute to isoform-specific functions such as differential localization [53]. At the extreme Cterminus, PKD1/2 contains a PDZ-domain binding motif (type I: X-(Ser/Thr)-X- $\phi$, where $\mathrm{X}$ is any amino acid and $\phi$ is a hydrophobic amino acid), which contains an autophosphorylation site [54]. The tail is likely also important in the regulation of PKD activity, since it has been shown that PKD1 C-terminal epitope tags increase in vitro autocatalytic activity and activity towards the peptide substrate syntide-2 compared to N-terminally tagged PKD1 [55].

\subsection{Activation Models of PKD}

2.2.1. Classical PKD Activation. In most instances, activation of PKD begins with diacylglycerol formation at membranes (e.g., after phospholipase $\mathrm{C}$ activation downstream of receptor tyrosine kinase or G-protein-coupled receptor activation, Figure 2(a)), although several exceptions have been discovered [56-68]. PKD binds to local pools of DAG via its C1 domains, which results in a conformational change, abrogating an autoinhibitory mechanism. At this stage, PKD expectedly autophosphorylates at the C-tail Ser-910 residue. This idea is supported by the fact that deletion of C1a and/or $\mathrm{C} 1 \mathrm{~b}$ in PKD1 results in an increased basal autocatalytic activity towards the Ser-910 autophosphorylation site and increased activity towards peptide substrate [69]. It is noteworthy that a deletion of the $\mathrm{C} 1$ domains does not increase basal activity towards protein substrates, nor in an increase of Ser-738/742 autophosphorylation in vitro [70]. Furthermore, Ser-910 phosphorylation does not require prior activation loop Ser-738/742 phosphorylation, since a S738/742A mutant still autophosphorylates Ser-910 while substrate phosphorylation is abolished [71]. This partially activated conformation likely allows PKCs (which colocalize at DAG-containing microenvironments via their respective $\mathrm{C} 1$ domains) to phosphorylate PKD at the activation loop Ser-738/742 residues. This phosphorylation will in turn stabilize a conformation in which the autoinhibition by the $\mathrm{PH}$ domain is relieved. This has been shown in a study by Waldron and Rozengurt where PKD1 bearing nonphosphorylatable Ser to Ala substitutions in the activation loop could not be activated, but when combined with a $\mathrm{PH}$ domain deletion (PKD1 S738/742A $\triangle \mathrm{PH}$ ), the kinase showed high basal activity towards Syntide-2. This activity could not be further stimulated with PDB in cellulo, an enzymatic profile that is comparable to PKD1 $\triangle \mathrm{PH}[72]$. This indicates that the role of activation loop phosphorylation is to stabilize the active conformation after the release of the $\mathrm{PH}$ domain. Indeed, in an isolated catalytic domain construct, Ser-738/742 substitution with Ala has a similar activity to that with a WT PKD1 catalytic domain construct [72]. This fully active PKD species will then act locally on substrates or relocate intracellularly to exert its function. All three isoforms can be activated by DAG in an activation loop phosphorylation-dependent manner. It should be noted however that there are differences in their regulation. For example, PKD3 does not contain a C-terminal Ser autophosphorylation site. Since it has been suggested that the phosphorylation of this site primes for subsequent autophosphorylation of the second Ser site in the activation loop (i.e., Ser-742) in PKD1, it is possible that PKD3 does not autophosphorylate at this residue [71]. Furthermore, the $\mathrm{C} 1$ domains of the different isoforms display different affinities for DAG [43], and a deletion of the C1 domains in $\mathrm{PKD} 2$ results in an inactivation rather than the activating effect seen for PKD1 [73], likely pointing to differences in their activation mechanisms.

2.2.2. PKD Activation in Oxidative Stress: An Isoform-Specific Matter. In oxidative stress conditions, the activation 


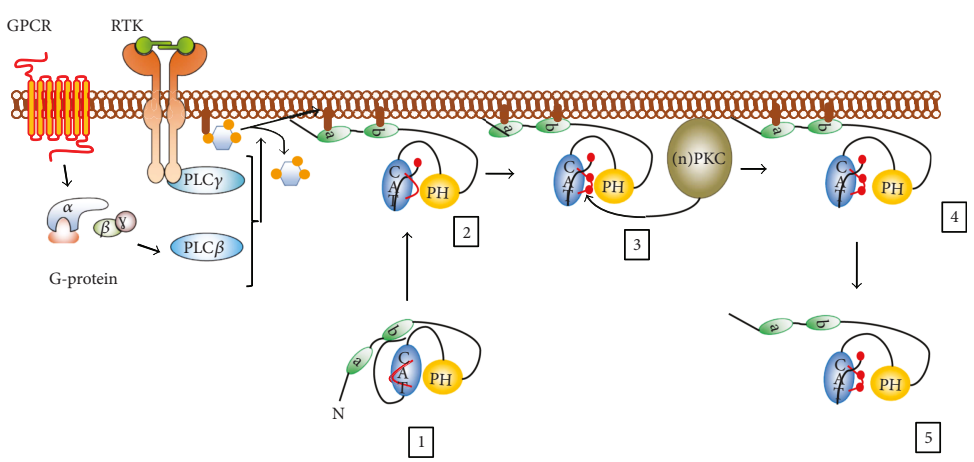

(a)

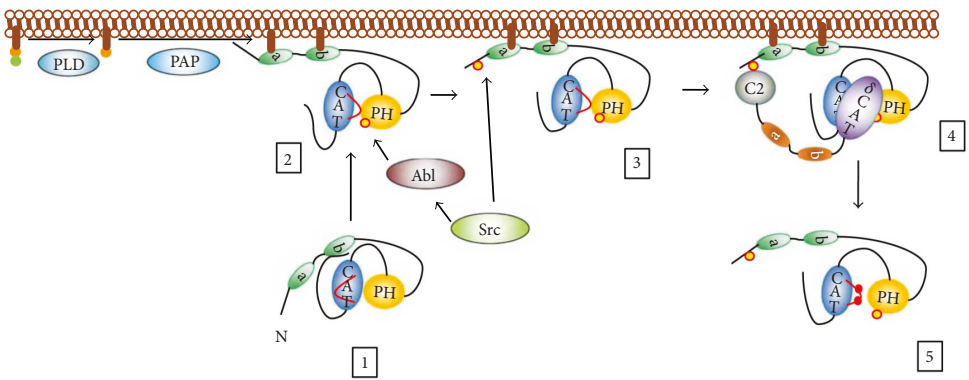

(b)

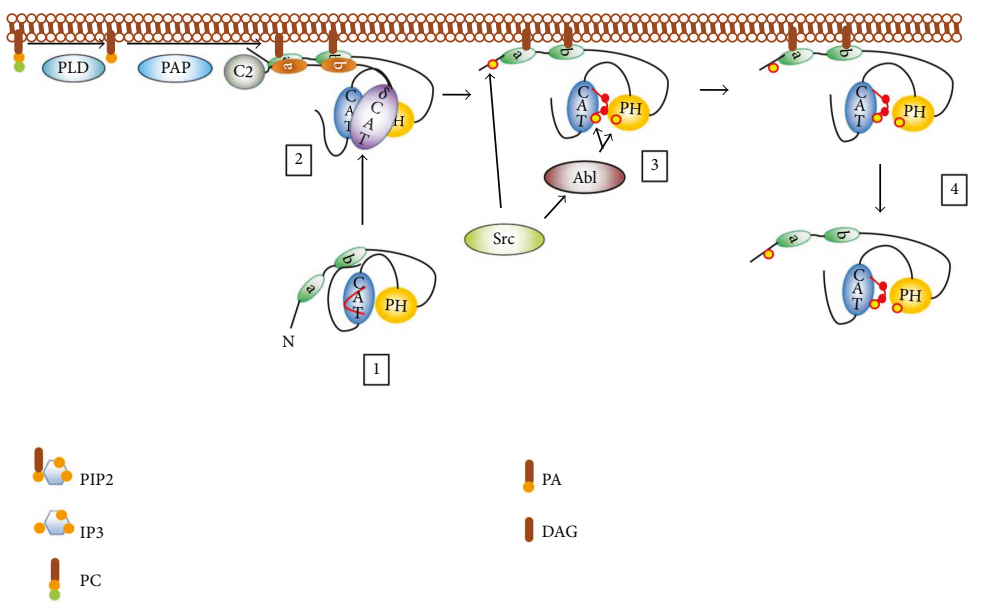

(c)

Figure 2: (a) Classical activation of PKD downstream of phospholipase C activity. (1) PKD1 is in an inactive resting conformation: the C1 and PH domains autoinhibit PKD activity. (2) PKD1 is recruited to DAG-containing microenvironments at the plasma membrane, which alleviates the autoinhibition exerted by the $\mathrm{C} 1$ domains. In this conformation, PKD has increased activity towards peptide substrates, but not towards proteins, indicative of an "unstable" open or "half-open" conformation. At this point, PKD can also exert autocatalytic activity towards Ser-910. (3) The abovementioned conformational changes and Ser-910 phosphorylation structure the kinase core for subsequent Ser-738 and Ser-742 phosphorylation by upstream PKCs. (4) Activation loop Ser phosphorylation stabilizes the PH-CAT module in an "open" conformation allowing for full PKD activity. (5) PKD1 is released from the membrane and translocates to several compartments to exert its functions. (b) Activation of PKD1 in oxidative stress conditions. (1) PKD1 is in a resting state, confer (a). (2) Activation is initiated by phosphorylation of Tyr-463 in the PH domain, which allows the recruitment of PKD to DAG generated by phospholipase D (PLD) activity at the outer mitochondrial membrane. (3) A subsequent conformational change allows for N-terminal phosphorylation at Tyr-95. (4) PKC $\delta$ docks to PKD1 via pTyr-95 and phosphorylates PKD1 at the activation loop Ser-738/742 residues. (5) PKD1 reaches full activity and initiates downstream signaling. (c) Activation of PKD2 in oxidative stress conditions. (1) PKD2 is in a resting state, confer (a). (2) PKD2 is recruited to DAG generated by phospholipase D (PLD) and phosphatidic acid phosphatase (PAP) activity at the outer mitochondrial membrane via its $\mathrm{C} 1$ domains, where it colocalizes and interacts with $\mathrm{PKC} \delta$ without the need for Tyr-95 phosphorylation. PKC $\delta$ phosphorylates PKD2 at the activation loop Ser-706/710 residues. (3) PKD2 is phosphorylated at Tyr residues, including Tyr-87, Tyr-438, and Tyr-717, with no determined hierarchy. (4) An active and Tyr-phosphorylated PKD2 species is released from the membrane to exert its functions. PIP2: phosphatidylinositol 4,5-bisphosphate; IP3: inositol 1,4,5-trisphosphate; PC: phosphatidylcholine; PA: phosphatidic acid; DAG: diacylglycerol. Tyr phosphorylation sites are indicated with yellow-coloured circles, and Ser phosphorylation sites are indicated with red-coloured circles. 
mechanism for PKD1 has historically been most studied and is well established. In contrast to classical activation by DAG downstream of G-protein-coupled receptors (GPCRs) or receptor tyrosine kinases (RTKs) through plasma membrane PLCs, activation of PKD1 in oxidative stress conditions is initiated by mitochondrial DAG production through phospholipase D (and phosphatidic acid phosphatase (PAP)) activity and generally requires the hierarchical phosphorylation of two Tyr residues in order to activate the kinase (Figures 1 and 2(b)) [74].

First, PKD1 is phosphorylated in the $\mathrm{PH}$ domain at Tyr-463 by Abl [75]. This initiates a conformational change, allowing for Src-mediated N-terminal phosphorylation at Tyr-95 [75, 76]. This residue is embedded in a motif that resembles the pTyr recognition motif for the $\mathrm{C} 2$ domain of PKC $\delta$. PKC $\delta$ docks to the pTyr motif and consequently phosphorylates the activation loop Ser-738/742 residues, an event shown to be crucial for PKD1 activity in oxidative stress (Figures 1 and 2(b)) [76-78]. In transformed cells, Ser-910 is found not to be phosphorylated in oxidative stress conditions and thus not part of the activation mechanism [77]. Recent data indicates some remarkable isoform-specific differences in the activation mechanism during oxidative stress. Indeed, in PKD2, Tyr phosphorylation does not prime for activation loop Ser-706/710 phosphorylation, but rather the opposite: activation loop Ser phosphorylation is necessary for subsequent Tyr phosphorylation of PKD2 (Figure 2(c)) [79]. How can this divergence be explained? A possible explanation is that in PKD1, phosphorylation of Tyr-463 in the $\mathrm{PH}$ domain is needed to promote the deinhibited state of the $\mathrm{PH}$ domain-catalytic domain interaction. This allows for subsequent activation loop Ser-738/742 phosphorylation, which further stabilizes the active conformation. N-terminal Tyr-95 phosphorylation is necessary to increase the affinity of PKD1 for PKC $\delta$ in this step. In PKD2, Tyr kinases likely have no access to the autoinhibited conformation. Activation of the kinase is in this case fully dependent on activation loop Ser-706/710 phosphorylation to stabilize the open conformation of the kinase after DAG binding. This active form of PKD2 can then be accessed by Tyr kinases. Rather than being involved in the activation of PKD2, the role of Tyr phosphorylation in the PKD2 PH domain could be to dock specific interactors in oxidative stress, since the $\mathrm{PH}$ domain in PKDs acts as a protein-protein interaction hub, and the sequence surrounding the Tyr residue is in agreement with the PTB domain consensus sequence NXXY [80]. N-terminal phosphorylation in PKD2 on the other hand could be beneficial to stabilize the PKD2-PKC $\delta$ interaction. It should be noted however that there is no difference in the association with $\mathrm{PKC} \delta$ between WT PKD2 and an N-terminal Tyr-Phe substituted mutant (PKD2 Y87F) during acute oxidative stress experiments [79]. This indicates that concentration of both kinases on DAG microenvironments on the outer mitochondrial membrane (OMM) can drive the interaction between PKC $\delta$ and PKD2 during acute stress, overruling the need for additional affinity contacts. However, Tyr-87 phosphorylation might be beneficial at regions of lower protein densities to increase the $\mathrm{PKC} \delta$-PKD2 affinity and to maintain PKD2 phosphorylation at the activation loop, for example, after dissociation from the OMM. In line with this, a recent study showed decreased activation loop phosphorylation of a PKD2 Y87F mutant when activated in focal adhesions [81]. Besides this differential activation mechanism, PKD2 is also differentially phosphorylated during oxidative stress. Indeed, PKD2 but not PKD1, is phosphorylated at Tyr in the $\mathrm{P}+1$ loop just before the APE motif. This is remarkable considering the fact that the activation segment is $100 \%$ conserved between the isoforms and highly conserved in all Ser/Thr kinases. This differential phosphorylation is due to a motif just C-terminal of the activation segment, which likely influences recognition by the upstream kinase c-Abl. This indicates a highly regulated signaling output towards the different isoforms in oxidative stress conditions. While no effects were seen on substrate selectivity on a peptide array, the phosphorylation of this site is shown to increase peptide substrate turnover in vitro [79].

PKD3 was long thought not to be activated in oxidative stress because it lacks an N-terminal Tyr residue. However, PKD3 was recently shown to be activated via oxidative stress in fibroblasts, which is reversible by treatment with the PKC inhibitor GF109203X [82]. This indicates that PKC can phosphorylate both PKD2 and PKD3 without Tyr phosphorylation at the $\mathrm{N}$-terminus during acute stress.

Besides isoform-specific behavior in oxidative stress conditions, certain cell-type-specific behavior was also observed. PKD1 activation in primary neuronal cells is associated with an increase in Ser-910 autophosphorylation (Figure 1) (this is in contrast with transformed cell lines) [83]. This effect has also been shown in another study in neuronal cells, where Ser-910 phosphorylation was shown to prime for activation loop phosphorylation of PKD1 [84]. Intriguingly, PKD1 activation does not involve Tyr phosphorylation in these studies $[83,84]$.

2.3. PKD Signaling in Oxidative Stress. Once activated, PKDs regulate several pathways downstream of oxidative stress. The best studied signaling output is towards the nuclear factor kappa-light-chain-enhancer of the activated $B$ cell $(\mathrm{NF}-\kappa \mathrm{B})$ pathway. PKD1 signals to NF- $\kappa \mathrm{B}$ via the IKK complex, which results in the consequential degradation of $\mathrm{I} \kappa \mathrm{B}$, but the exact mechanism has not been elucidated yet [85]. $\mathrm{NF}-\kappa \mathrm{B}$ activity results in upregulation of mnSOD, which detoxifies the cell from mROS, but also generates $\mathrm{H}_{2} \mathrm{O}_{2}$, a tumor-promoting signaling molecule [86]. Additionally, it was recently shown that ROS-induced PKD1-mediated $\mathrm{NF}-\kappa \mathrm{B}$ activity results in the upregulation of epidermal growth factor receptor (EGFR) signaling components (EGFR and its ligand $\mathrm{TGF} \alpha$ ) in pancreatic cancer downstream of oncogenic Ras [87]. While PKD-induced NF- $\kappa \mathrm{B}$ activity in transformed cells contributes to tumor development, this pathway has recently also been shown to contribute to the physiological steady-state survival of neuronal cells. Indeed, in these cells, the PKD-NF- $\kappa \mathrm{B}-\mathrm{SOD} 2$ axis is constitutively active and protects against oxidative damage [88]. In a model of excitotoxic neurodegeneration, which results in endoplasmic reticulum stress and mitochondrial dysfunction, high levels of ROS, and oxidative stress damage, the authors 

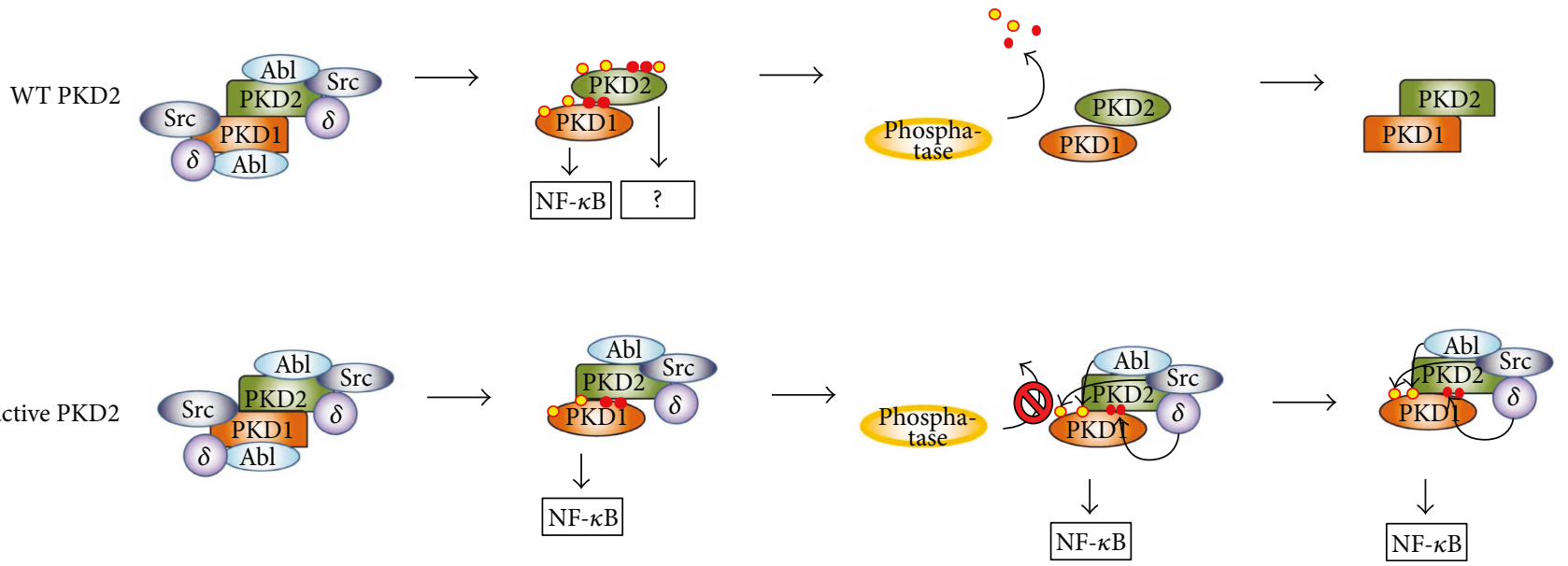

FIgURE 3: Putative function of PKD2 in NF- $\kappa \mathrm{B}$ signaling, based on observations with inactive PKD2. Upon exposure to oxidative stress, a kinase complex comprising dimeric PKD1/2, Src, Abl, and $\mathrm{PKC} \delta$ is assembled on the outer mitochondrial membrane. In a WT PKD1/2 dimer, the upstream kinases phosphorylate PKD1/2, which causes loss of affinity and consequentially the release of upstream kinases. PKD1 and PKD2 are both activated, but only PKD1 signals to NF- $\kappa$ B. When PKD2 cannot be phosphorylated because of mutations, the upstream kinases retain their affinity for PKD2 and can keep PKD1 phosphorylated (since they will have a similar affinity for PKD1 when it is dephosphorylated). In conjunction with this, the inactive conformation of PKD2, resulting from the fact that it cannot be phosphorylated, could protect PKD1 from phosphatase action. Both these phenomena could explain the enhanced NF- $\kappa \mathrm{B}$ signaling output seen with inactive PKD2 mutants. Tyr phosphorylation sites are indicated with yellow-coloured circles, and Ser phosphorylation sites are indicated with red-coloured circles.

showed that PKD1 is rapidly deactivated after a short burst of activity, resulting in the loss of NF- $\kappa \mathrm{B}$ signaling and impeding neuronal survival [88]. These findings were further substantiated in vivo using patient samples and an ischemic mouse model [88]. Another recent study in hippocampal primary neurons showed that PKD1 is transiently activated during oxidative stress, but no changes in NF- $\kappa \mathrm{B}$ signaling were observed by the authors [83]. Notably, while activity and activation loop Ser-738/742 phosphorylation are necessary for signaling output to $\mathrm{NF}-\kappa \mathrm{B}$ by PKD1, for PKD2, the inverse has been shown $[77,89]$. Indeed, a kinase-dead PKD2 D695A (DFG to AFG) mutant strongly stimulates NF- $\kappa$ B signaling, as do an inactivated S706/710A or Y717F mutants, while WT PKD2 (i.e., activated) or an activation loop phosphomimetic S706/710E mutant displays no increased signaling output in oxidative stress conditions $[79,89]$. The reason for this isoform-specific behavior in oxidative stress is not known. One possibility is that PKD2 itself does not signal to NF- $\kappa \mathrm{B}$ but acts as a scaffold for PKD1, which phosphorylates an isoform-specific substrate in the NF- $\kappa$ B pathway. Indeed, PKD1 and PKD2 are known to form heterodimers [90]. The fact that PKD2 cannot be phosphorylated by its upstream kinases through S706/710A or Y717F mutations could "trap" the upstream kinase complex (i.e., $\mathrm{PKC} \delta, \mathrm{Abl}$, and potentially Src) on inactive PKD2. Due to dimer formation of PKD2 with PKD1, the latter is kept phosphorylated by the upstream kinases held in proximity by $\mathrm{PKD} 2$, potentiating its activity on NF- $\kappa \mathrm{B}$ (Figure 3 ). In addition, the inactive conformation of the PKD2 monomer in the heterodimer could protect PKD1 from phosphatases (Figure 3). It should be noted however that it is unknown whether an Asp-Ala substitution in the DFG motif of the PKD2 activation segment has an effect on the ability of PKC $\delta$ and Abl to phosphorylate Ser-706/710 and Tyr-717 residues. Additionally, PKD2 can potentially enhance PKD1-mediated signaling by recruiting $\mathrm{NF}-\kappa \mathrm{B}$ signaling components via its $\mathrm{PH}$ domain in a Tyr-438 phosphorylation-dependent manner. Indeed, a phosphomimetic mutant of this residue displays increased signaling output to NF- $\kappa \mathrm{B}$ in the absence of $\mathrm{H}_{2} \mathrm{O}_{2}$ when combined with an inactivating D695A mutation, but not when combined with a S706/710E mutant [89].

Besides the effects of PKDs on NF- $\kappa$ B, other PKDdependent signaling functions have been described. For example, PKD1 inhibits mitochondrial depolarization and decreases cytochrome $c$ release upon oxidative stress in mouse embryonic fibroblasts, effectively protecting them from apoptosis [91]. Interestingly, this behavior is isoformspecific, since cells expressing PKD2/3 do not display this phenotype. In hepatocytes, PKD1 has also been shown to protect cells from apoptosis by downregulating JNK signaling [92]. In epithelioid RIE-1 cells, PKD1 not only signals to NF- $\kappa$ B but also reduces p38 phosphorylation, both of which contribute to protection from apoptosis [93]. No alterations were seen in JNK signaling in this context [93]. PKD1 also phosphorylates $\mathrm{Hsp} 27$ in response to oxidative stress $[94,95]$. In neuronal cells, Hsp27 phosphorylation protects from ischemia-induced apoptosis by suppressing JNK activity [95].

Notably, all of the PKD1 functions described above result in prosurvival signals in oxidative stress. However, in a PKCindependent pathway, oxidative stress-induced PKD1 activity can also activate JNK downstream of death-associated 
protein kinase (DAPK), which results in a prodeath signal and increased necrotic cell death [96]. Furthermore, in bovine aortic ECs (BAECs), PKD1 has been shown to activate JNK via association with its upstream kinase ASK1. This association is dependent on 14-3-3 binding to PKD1, potentially via Ser-205/208 and Ser-219/223 autophosphorylation (Figure 1). The consequence of JNK activation in these cells has not been explored [44]. PKD1 also has been shown to phosphorylate Vps34 in oxidative stress [97]. Vps34 is a PI3 kinase and its phosphorylation by PKD1 leads to an activation and consequential increase of $\mathrm{PI}(3) \mathrm{P}$, resulting in increased autophagy and presumably cell death [97].

\section{Concluding Remarks and Perspectives}

The activation, regulation, and signaling properties of protein kinase $\mathrm{D}$ isoforms in oxidative stress come with intriguing questions that require further exploration. The remarkable isoform-specific differences point to a highly specific regulation of these kinases. Isoform-specific behavior in oxidative stress is also seen for other kinase families. For example, Akt isoforms are differentially regulated in oxidative stress, where Akt2 is specifically inactivated after $\mathrm{H}_{2} \mathrm{O}_{2}$ stimulation via the generation of a disulfide bond [98]. Besides their differential regulation, PKD isoforms also display distinct signaling properties. A striking example of this is their different signaling output to $\mathrm{NF}-\kappa \mathrm{B}$. While activated PKD1 in oxidative stress signals to NF- $\kappa \mathrm{B}$, it has been shown that for $\mathrm{PKD} 2$, inactive forms stimulate the NF- $\kappa \mathrm{B}$ signaling output $[79,89]$. However, the functional relevance of inactive PKD2 is unclear, since it is also activated during oxidative stress, likely to an even larger extent than PKD1 [79]. The role of WT PKD2 in NF- $\kappa \mathrm{B}$ signaling as part of a PKD1PKD2 heterodimer is likely twofold: (1) to recruit NF- $\kappa \mathrm{B}$ signaling components via its $\mathrm{PH}$ domain and (2) to enhance complex formation with upstream kinases to enhance PKD1 activation. An important question is whether there are other, currently unknown pathways activated PKD2 contributes to in oxidative stress.

Isoform-specific signaling behavior in redox signaling is also observed within the PKC family. For example, in the protection of the heart from ischemic events, the related nPKCs $\mathrm{PKC} \delta$ and $\mathrm{PKC} \varepsilon$ play opposing roles, with $\mathrm{PKC} \varepsilon$ being cardioprotective while $\mathrm{PKC} \delta$ increases damage induced by ischemia both in vitro and in vivo [99].

Another level of complexity lies within cell-type-specific behavior of PKDs in oxidative stress, both in their regulation and in their signaling properties. For example, as mentioned before, in neuronal cells, PKD1 activation does not always involve Tyr phosphorylation, but rather Ser-910 phosphorylation, and it does not contribute to an increased NF- $\kappa \mathrm{B}$ signaling output in these cells $[83,84]$. Moreover, a recent study shows a loss of homeostatic NF- $\kappa$ B signaling output during increased oxidative stress due to a rapid downregulation of PKD1 activity [88].

In conclusion, future studies should be carefully carried out to dissect the ROS-mediated regulation and functional roles of the individual PKD isoforms in different cell types, in order to understand the full extent of PKD-mediated signaling in oxidative stress.

\section{Disclosure}

Mathias Cobbaut's present address is The Francis Crick Institute, London, United Kingdom.

\section{Conflicts of Interest}

The authors declare that there is no conflict of interest regarding the publication of this paper.

\section{References}

[1] C. L. Quinlan, J. R. Treberg, I. V. Perevoshchikova, A. L. Orr, and M. D. Brand, "Native rates of superoxide production from multiple sites in isolated mitochondria measured using endogenous reporters," Free Radical Biology \& Medicine, vol. 53, no. 9, pp. 1807-1817, 2012.

[2] M. Schrader and H. D. Fahimi, "Peroxisomes and oxidative stress," Biochimica et Biophysica Acta (BBA) - Molecular Cell Research, vol. 1763, no. 12, pp. 1755-1766, 2006.

[3] B. Bhandary, A. Marahatta, H. R. Kim, and H. J. Chae, "An involvement of oxidative stress in endoplasmic reticulum stress and its associated diseases," International Journal of Molecular Sciences, vol. 14, no. 1, pp. 434-456, 2013.

[4] B. Rada and T. L. Leto, "Oxidative innate immune defenses by Nox/Duox family NADPH oxidases," Contributions to Microbiology, vol. 15, pp. 164-187, 2008.

[5] M. Sundaresan, Z. X. Yu, V. J. Ferrans, K. Irani, and T. Finkel, "Requirement for generation of $\mathrm{H}_{2} \mathrm{O}_{2}$ for platelet-derived growth factor signal transduction," Science, vol. 270, no. 5234, pp. 296-299, 1995.

[6] Y. S. Bae, S. W. Kang, M. S. Seo et al., "Epidermal growth factor (EGF)-induced generation of hydrogen peroxide. Role in EGF receptor-mediated tyrosine phosphorylation," The Journal of Biological Chemistry, vol. 272, no. 1, pp. 217-221, 1997.

[7] S. R. Lee, K. S. Yang, J. Kwon, C. Lee, W. Jeong, and S. G. Rhee, "Reversible inactivation of the tumor suppressor PTEN by $\mathrm{H}_{2} \mathrm{O}_{2}$," The Journal of Biological Chemistry, vol. 277, no. 23, pp. 20336-20342, 2002.

[8] E. Giannoni, F. Buricchi, G. Raugei, G. Ramponi, and P. Chiarugi, "Intracellular reactive oxygen species activate Src tyrosine kinase during cell adhesion and anchoragedependent cell growth," Molecular and Cellular Biology, vol. 25, no. 15, pp. 6391-6403, 2005.

[9] A. W. Girotti, "Mechanisms of lipid peroxidation," Journal of Free Radicals in Biology \& Medicine, vol. 1, no. 2, pp. 87-95, 1985.

[10] M. S. Cooke, M. D. Evans, M. Dizdaroglu, and J. Lunec, "Oxidative DNA damage: mechanisms, mutation, and disease," The FASEB Journal, vol. 17, no. 10, pp. 1195-1214, 2003.

[11] A. L. Jackson, R. Chen, and L. A. Loeb, "Induction of microsatellite instability by oxidative DNA damage," Proceedings of the National Academy of Sciences of the United States of America, vol. 95, no. 21, pp. 12468-12473, 1998.

[12] R. Ghosh and D. L. Mitchell, "Effect of oxidative DNA damage in promoter elements on transcription factor binding," Nucleic Acids Research, vol. 27, no. 15, pp. 3213-3218, 1999. 
[13] E. Cadenas, "Mitochondrial free radical production and cell signaling," Molecular Aspects of Medicine, vol. 25, no. 1-2, pp. 17-26, 2004.

[14] H. U. Simon, A. Haj-Yehia, and F. Levi-Schaffer, "Role of reactive oxygen species (ROS) in apoptosis induction," Apoptosis, vol. 5, no. 5, pp. 415-418, 2000.

[15] K. J. Davies, "Protein damage and degradation by oxygen radicals. I. General aspects," The Journal of Biological Chemistry, vol. 262, no. 20, pp. 9895-9901, 1987.

[16] E. R. Stadtman, "Metal ion-catalyzed oxidation of proteins: biochemical mechanism and biological consequences," Free Radical Biology \& Medicine, vol. 9, no. 4, pp. 315-325, 1990.

[17] S. Gromer, S. Urig, and K. Becker, "The thioredoxin systemfrom science to clinic," Medicinal Research Reviews, vol. 24, no. 1, pp. 40-89, 2004.

[18] S. Curello, C. Ceconi, C. Bigoli, R. Ferrari, A. Albertini, and C. Guarnieri, "Changes in the cardiac glutathione status after ischemia and reperfusion," Experientia, vol. 41, no. 1, pp. 4243, 1985 .

[19] D. Sheehan, G. Meade, V. M. Foley, and C. A. Dowd, "Structure, function and evolution of glutathione transferases: implications for classification of non-mammalian members of an ancient enzyme superfamily," Biochemical Journal, vol. 360, no. 1, pp. 1-16, 2001.

[20] H. Sies, "Glutathione and its role in cellular functions," Free Radical Biology \& Medicine, vol. 27, no. 9-10, pp. 916-921, 1999.

[21] N. S. Dhalla, R. M. Temsah, and T. Netticadan, "Role of oxidative stress in cardiovascular diseases," Journal of Hypertension, vol. 18, no. 6, pp. 655-673, 2000.

[22] D. Kienhofer, S. Boeltz, and M. H. Hoffmann, "Reactive oxygen homeostasis - the balance for preventing autoimmunity," Lupus, vol. 25, no. 8, pp. 943-954, 2016.

[23] K. Richter and T. Kietzmann, "Reactive oxygen species and fibrosis: further evidence of a significant liaison," Cell and Tissue Research, vol. 365, no. 3, pp. 591-605, 2016.

[24] A. Popa-Wagner, S. Mitran, S. Sivanesan, E. Chang, and A. M. Buga, "ROS and brain diseases: the good, the bad, and the ugly," Oxidative Medicine and Cellular Longevity, vol. 2013, Article ID 963520, 14 pages, 2013.

[25] G. Y. Liou and P. Storz, "Reactive oxygen species in cancer," Free Radical Research, vol. 44, no. 5, pp. 479-496, 2010.

[26] T. P. Szatrowski and C. F. Nathan, "Production of large amounts of hydrogen peroxide by human tumor cells," Cancer Research, vol. 51, no. 3, pp. 794-798, 1991.

[27] R. A. Cairns, I. S. Harris, and T. W. Mak, "Regulation of cancer cell metabolism," Nature Reviews Cancer, vol. 11, no. 2, pp. 85-95, 2011.

[28] A. Chatterjee, E. Mambo, and D. Sidransky, "Mitochondrial DNA mutations in human cancer," Oncogene, vol. 25, no. 34, pp. 4663-4674, 2006.

[29] H. C. Lee, P. H. Yin, J. C. Lin et al., "Mitochondrial genome instability and mtDNA depletion in human cancers," Annals of the New York Academy of Sciences, vol. 1042, no. 1, pp. 109-122, 2005.

[30] E. Mambo, A. Chatterjee, M. Xing et al., "Tumor-specific changes in mtDNA content in human cancer," International Journal of Cancer, vol. 116, no. 6, pp. 920-924, 2005.

[31] K. Irani, Y. Xia, J. L. Zweier et al., "Mitogenic signaling mediated by oxidants in Ras-transformed fibroblasts," Science, vol. 275, no. 5306, pp. 1649-1652, 1997.
[32] U. Weyemi, O. Lagente-Chevallier, M. Boufraqech et al., "ROS-generating NADPH oxidase NOX4 is a critical mediator in oncogenic H-Ras-induced DNA damage and subsequent senescence," Oncogene, vol. 31, no. 9, pp. 1117-1129, 2012.

[33] O. Vafa, M. Wade, S. Kern et al., "c-Myc can induce DNA damage, increase reactive oxygen species, and mitigate p53 function: a mechanism for oncogene-induced genetic instability," Molecular Cell, vol. 9, no. 5, pp. 1031-1044, 2002.

[34] A. Maya-Mendoza, J. Ostrakova, M. Kosar et al., "Myc and Ras oncogenes engage different energy metabolism programs and evoke distinct patterns of oxidative and DNA replication stress," Molecular Oncology, vol. 9, no. 3, pp. 601-616, 2015.

[35] C. W. Lin, L. Y. Yang, S. C. Shen, and Y. C. Chen, "IGF-I plus E2 induces proliferation via activation of ROS-dependent ERKs and JNKs in human breast carcinoma cells," Journal of Cellular Physiology, vol. 212, no. 3, pp. 666-674, 2007.

[36] L. Z. Liu, X. W. Hu, C. Xia et al., "Reactive oxygen species regulate epidermal growth factor-induced vascular endothelial growth factor and hypoxia-inducible factor- $1 \alpha$ expression through activation of AKT and P70S6K1 in human ovarian cancer cells," Free Radical Biology \& Medicine, vol. 41, no. 10, pp. 1521-1533, 2006.

[37] N. R. Leslie, D. Bennett, Y. E. Lindsay, H. Stewart, A. Gray, and C. P. Downes, "Redox regulation of PI 3-kinase signalling via inactivation of PTEN," The EMBO Journal, vol. 22, no. 20, pp. 5501-5510, 2003.

[38] E. Rozengurt, O. Rey, and R. T. Waldron, "Protein kinase D signaling," The Journal of Biological Chemistry, vol. 280, no. 14, pp. 13205-13208, 2005.

[39] V. Malhotra and F. Campelo, "PKD regulates membrane fission to generate TGN to cell surface transport carriers," Cold Spring Harbor Perspectives in Biology, vol. 3, no. 2, 2011.

[40] F. J. Johannes, J. Prestle, S. Eis, P. Oberhagemann, and K. Pfizenmaier, "PKCu is a novel, atypical member of the protein kinase C family," The Journal of Biological Chemistry, vol. 269, no. 8, pp. 6140-6148, 1994.

[41] E. Rozengurt, J. Sinnett-Smith, J. Van Lint, and A. M. Valverde, "Protein kinase D (PKD): a novel target for diacylglycerol and phorbol esters," Mutation Research, vol. 333, no. 1-2, pp. $153-$ 160, 1995.

[42] J. V. Van Lint, J. Sinnett-Smith, and E. Rozengurt, "Expression and characterization of PKD, a phorbol ester and diacylglycerol-stimulated serine protein kinase," The Journal of Biological Chemistry, vol. 270, no. 3, pp. 1455-1461, 1995.

[43] J. Chen, F. Deng, J. Li, and Q. J. Wang, "Selective binding of phorbol esters and diacylglycerol by individual $\mathrm{C} 1$ domains of the PKD family," The Biochemical Journal, vol. 411, no. 2, pp. 333-342, 2008.

[44] W. Zhang, S. Zheng, P. Storz, and W. Min, "Protein kinase D specifically mediates apoptosis signal-regulating kinase 1-JNK signaling induced by $\mathrm{H}_{2} \mathrm{O}_{2}$ but not tumor necrosis factor," The Journal of Biological Chemistry, vol. 280, no. 19, pp. 19036-19044, 2005.

[45] M. Gschwendt, F. J. Johannes, W. Kittstein, and F. Marks, "Regulation of protein kinase Cmu by basic peptides and heparin. Putative role of an acidic domain in the activation of the kinase," The Journal of Biological Chemistry, vol. 272, no. 33, pp. 20742-20746, 1997.

[46] T. Iglesias and E. Rozengurt, "Protein kinase D activation by mutations within its pleckstrin homology domain," The 
Journal of Biological Chemistry, vol. 273, no. 1, pp. 410-416, 1998.

[47] M. A. Lemmon, "Pleckstrin homology domains: not just for phosphoinositides," Biochemical Society Transactions, vol. 32, no. 5, pp. 707-711, 2004.

[48] W. S. Park, W. D. Heo, J. H. Whalen et al., "Comprehensive identification of PIP3-regulated PH domains from C. elegans to $H$. sapiens by model prediction and live imaging," Molecular Cell, vol. 30, no. 3, pp. 381-392, 2008.

[49] K. Scheffzek and S. Welti, "Pleckstrin homology (PH) like domains - versatile modules in protein-protein interaction platforms," FEBS Letters, vol. 586, no. 17, pp. 2662-2673, 2012.

[50] C. Jamora, N. Yamanouye, J. Van Lint et al., “ $G \beta \gamma$-mediated regulation of Golgi organization is through the direct activation of protein kinase D," Cell, vol. 98, no. 1, pp. 59-68, 1999.

[51] W. W. Lau, A. S. Chan, L. S. Poon, J. Zhu, and Y. H. Wong, "G $\beta \gamma$-mediated activation of protein kinase D exhibits subunit specificity and requires $\mathrm{G} \beta \gamma$-responsive phospholipase $\mathrm{C} \beta$ isoforms," Cell Communication and Signaling, vol. 11, no. 1, p. 22, 2013.

[52] R. T. Waldron, O. Rey, T. Iglesias, T. Tugal, D. Cantrell, and E. Rozengurt, "Activation loop Ser744 and Ser748 in protein kinase D are transphosphorylated in vivo," The Journal of Biological Chemistry, vol. 276, no. 35, pp. 32606-32615, 2001.

[53] R. Papazyan, E. Rozengurt, and O. Rey, "The C-terminal tail of protein kinase D2 and protein kinase D3 regulates their intracellular distribution," Biochemical and Biophysical Research Communications, vol. 342, no. 3, pp. 685-689, 2006.

[54] L. Sanchez-Ruiloba, N. Cabrera-Poch, M. Rodriguez-Martinez et al., "Protein kinase D intracellular localization and activity control kinase D-interacting substrate of $220-\mathrm{kDa}$ traffic through a postsynaptic density-95/discs large/zonula occludens-1-binding motif," The Journal of Biological Chemistry, vol. 281, no. 27, pp. 18888-18900, 2006.

[55] W. Qiu, F. Zhang, and S. F. Steinberg, "The protein kinase D1 $\mathrm{COOH}$ terminus: marker or regulator of enzyme activity?," American Journal of Physiology Cell physiology, vol. 307, no. 7, pp. C606-C610, 2014.

[56] M. S. Kim, F. Wang, P. Puthanveetil et al., "Cleavage of protein kinase D after acute hypoinsulinemia prevents excessive lipoprotein lipase-mediated cardiac triglyceride accumulation," Diabetes, vol. 58, no. 11, pp. 2464-2475, 2009.

[57] T. Vantus, D. Vertommen, X. Saelens et al., "Doxorubicininduced activation of protein kinase D1 through caspasemediated proteolytic cleavage: identification of two cleavage sites by microsequencing," Cellular Signalling, vol. 16, no. 6 , pp. 703-709, 2004.

[58] J. Lemonnier, C. Ghayor, J. Guicheux, and J. Caverzasio, "Protein kinase C-independent activation of protein kinase D is involved in BMP-2-induced activation of stress mitogenactivated protein kinases JNK and p38 and osteoblastic cell differentiation," The Journal of Biological Chemistry, vol. 279, no. 1, pp. 259-264, 2003.

[59] W. M. Cheung, W. W. Ng, and A. W. Kung, "Dimethyl sulfoxide as an inducer of differentiation in preosteoblast MC3T3-E1 cells," FEBS Letters, vol. 580, no. 1, pp. 121-126, 2006.

[60] D. Y. Kim, K. H. Park, M. S. Jung et al., "Ginsenoside Rh2(S) induces differentiation and mineralization of MC3T3-E1 cells through activation of the PKD/AMPK signaling pathways," International Journal of Molecular Medicine, vol. 28, no. 5, pp. 753-759, 2011.
[61] M. D. Bradford and S. P. Soltoff, "P2X7 receptors activate protein kinase D and p42/p44 mitogen-activated protein kinase (MAPK) downstream of protein kinase C," The Biochemical Journal, vol. 366, no. 3, pp. 745-755, 2002.

[62] J. E. Park, Y. I. Kim, and A. K. Yi, "Protein kinase D1: a new component in TLR9 signaling," Journal of Immunology, vol. 181, no. 3, pp. 2044-2055, 2008.

[63] F. Rezaee, N. Meednu, J. A. Emo et al., "Polyinosinic: polycytidylic acid induces protein kinase D-dependent disassembly of apical junctions and barrier dysfunction in airway epithelial cells," The Journal of Allergy and Clinical Immunology, vol. 128, no. 1216-1224, article e11, 2011.

[64] J. Yuan, L. W. Slice, and E. Rozengurt, "Activation of protein kinase $\mathrm{D}$ by signaling through Rho and the $\alpha$ subunit of the heterotrimeric G protein G13," The Journal of Biological Chemistry, vol. 276, no. 42, pp. 38619-38627, 2001.

[65] T. Eiseler, H. Doppler, I. K. Yan, K. Kitatani, K. Mizuno, and P. Storz, "Protein kinase D1 regulates cofilin-mediated F-actin reorganization and cell motility through slingshot," Nature Cell Biology, vol. 11, no. 5, pp. 545-556, 2009.

[66] H. Doppler, L. I. Bastea, S. Borges, S. J. Spratley, S. E. Pearce, and P. Storz, "Protein kinase D isoforms differentially modulate cofilin-driven directed cell migration," PLoS One, vol. 9, no. 5, article e98090, 2014.

[67] G. V. Pusapati, T. Eiseler, A. Rykx et al., "Protein kinase D regulates RhoA activity via rhotekin phosphorylation," The Journal of Biological Chemistry, vol. 287, no. 12, pp. 94739483, 2012.

[68] V. McEneaney, B. J. Harvey, and W. Thomas, "Aldosterone rapidly activates protein kinase $\mathrm{D}$ via a mineralocorticoid receptor/EGFR trans-activation pathway in the M1 kidney CCD cell line," The Journal of Steroid Biochemistry and Molecular Biology, vol. 107, no. 3-5, pp. 180-190, 2007.

[69] T. Iglesias and E. Rozengurt, "Protein kinase D activation by deletion of its cysteine-rich motifs," FEBS Letters, vol. 454, no. 1-2, pp. 53-56, 1999.

[70] V. O. Rybin, J. Guo, E. Harleton, F. Zhang, and S. F. Steinberg, "Regulatory domain determinants that control PKD1 activity," The Journal of Biological Chemistry, vol. 287, no. 27, pp. 22609-22615, 2012.

[71] V. O. Rybin, J. Guo, and S. F. Steinberg, "Protein kinase D1 autophosphorylation via distinct mechanisms at Ser744/ Ser748 and Ser916," The Journal of Biological Chemistry, vol. 284, no. 4, pp. 2332-2343, 2009.

[72] R. T. Waldron and E. Rozengurt, "Protein kinase C phosphorylates protein kinase D activation loop Ser744 and Ser748 and releases autoinhibition by the pleckstrin homology domain," The Journal of Biological Chemistry, vol. 278, no. 1, pp. 154163, 2003.

[73] A. Auer, J. von Blume, S. Sturany et al., "Role of the regulatory domain of protein kinase D2 in phorbol ester binding, catalytic activity, and nucleocytoplasmic shuttling," Molecular Biology of the Cell, vol. 16, no. 9, pp. 4375-4385, 2005.

[74] C. F. Cowell, H. Doppler, I. K. Yan, A. Hausser, Y. Umezawa, and P. Storz, "Mitochondrial diacylglycerol initiates proteinkinase D1-mediated ROS signaling," Journal of Cell Science, vol. 122, no. 7, pp. 919-928, 2009.

[75] P. Storz, H. Doppler, F. J. Johannes, and A. Toker, “Tyrosine phosphorylation of protein kinase $\mathrm{D}$ in the pleckstrin homology domain leads to activation," The Journal of Biological Chemistry, vol. 278, no. 20, pp. 17969-17976, 2003. 
[76] H. Doppler and P. Storz, “A novel tyrosine phosphorylation site in protein kinase $\mathrm{D}$ contributes to oxidative stressmediated activation," The Journal of Biological Chemistry, vol. 282, no. 44, pp. 31873-31881, 2007.

[77] P. Storz, H. Doppler, and A. Toker, “Activation loop phosphorylation controls protein kinase D-dependent activation of nuclear factor $\kappa \mathrm{B}$," Molecular Pharmacology, vol. 66, no. 4, pp. 870-879, 2004.

[78] R. T. Waldron and E. Rozengurt, "Oxidative stress induces protein kinase $\mathrm{D}$ activation in intact cells. Involvement of Src and dependence on protein kinase C," The Journal Biological Chemistry, vol. 275, no. 22, pp. 17114-17121, 2000.

[79] M. Cobbaut, R. Derua, H. Doppler et al., "Differential regulation of PKD isoforms in oxidative stress conditions through phosphorylation of a conserved Tyr in the $\mathrm{P}+1$ loop," Scientific Reports, vol. 7, no. 1, p. 887, 2017.

[80] M. J. Smith, W. R. Hardy, J. M. Murphy, N. Jones, and T. Pawson, "Screening for PTB domain binding partners and ligand specificity using proteome-derived NPXY peptide arrays," Molecular and Cellular Biology, vol. 26, no. 22, pp. 8461-8474, 2006.

[81] N. Durand, L. I. Bastea, H. Doppler, T. Eiseler, and P. Storz, "Src-mediated tyrosine phosphorylation of protein kinase D2 at focal adhesions regulates cell adhesion," Scientific Reports, vol. 7, no. 1, p. 9524, 2017.

[82] W. Qiu and S. F. Steinberg, "Phos-tag SDS-PAGE resolves agonist- and isoform-specific activation patterns for PKD2 and PKD3 in cardiomyocytes and cardiac fibroblasts," Journal of Molecular and Cellular Cardiology, vol. 99, pp. 14-22, 2016.

[83] H. Liliom, K. Tarnok, Z. Abraham, B. Racz, A. Hausser, and K. Schlett, "Protein kinase D exerts neuroprotective functions during oxidative stress via nuclear factor kappa Bindependent signaling pathways," Journal of Neurochemistry, vol. 142, no. 6, pp. 948-961, 2017.

[84] A. Asaithambi, A. Kanthasamy, H. Saminathan, V. Anantharam, and A. G. Kanthasamy, "Protein kinase D1 (PKD1) activation mediates a compensatory protective response during early stages of oxidative stress-induced neuronal degeneration," Molecular Neurodegeneration, vol. 6, no. 1, p. 43, 2011.

[85] P. Storz and A. Toker, "Protein kinase D mediates a stressinduced NF- $\kappa \mathrm{B}$ activation and survival pathway," The EMBO Journal, vol. 22, no. 1, pp. 109-120, 2003.

[86] P. Storz, H. Doppler, and A. Toker, "Protein kinase D mediates mitochondrion-to-nucleus signaling and detoxification from mitochondrial reactive oxygen species," Molecular and Cellular Biology, vol. 25, no. 19, pp. 8520-8530, 2005.

[87] G. Y. Liou, H. Doppler, K. E. DelGiorno et al., "Mutant KRas-induced mitochondrial oxidative stress in acinar cells upregulates EGFR signaling to drive formation of pancreatic precancerous lesions," Cell Reports, vol. 14, no. 10, pp. 2325-2336, 2016.

[88] J. Pose-Utrilla, L. Garcia-Guerra, A. Del Puerto et al., "Excitotoxic inactivation of constitutive oxidative stress detoxification pathway in neurons can be rescued by PKD1," Nature Communications, vol. 8, no. 1, p. 2275, 2017.

[89] T. Mihailovic, M. Marx, A. Auer et al., "Protein kinase D2 mediates activation of nuclear factor $\kappa \mathrm{B}$ by Bcr-Abl in Bcr$\mathrm{Abl}^{+}$human myeloid leukemia cells," Cancer Research, vol. 64, no. 24, pp. 8939-8944, 2004.
[90] C. Aicart-Ramos, S. D. He, M. Land, and C. S. Rubin, "A novel conserved domain mediates dimerization of protein kinase D (PKD) isoforms: dimerization is essential for PKD-dependent regulation of secretion and innate immunity," The Journal of Biological Chemistry, vol. 291, no. 45, pp. 23516-23531, 2016.

[91] T. Zhang, P. Sell, U. Braun, and M. Leitges, "PKD1 protein is involved in reactive oxygen species-mediated mitochondrial depolarization in cooperation with protein kinase $\mathrm{C} \delta$ (PKC 8$)$," The Journal of Biological Chemistry, vol. 290, no. 16, pp. 10472-10485, 2015.

[92] Y. Wang, J. M. Schattenberg, R. M. Rigoli, P. Storz, and M. J. Czaja, "Hepatocyte resistance to oxidative stress is dependent on protein kinase C-mediated down-regulation of c-Jun/ AP-1," The Journal of Biological Chemistry, vol. 279, no. 30, pp. 31089-31097, 2004.

[93] J. Song, J. Li, J. Qiao, S. Jain, B. Mark Evers, and D. H. Chung, "PKD prevents $\mathrm{H}_{2} \mathrm{O}_{2}$-induced apoptosis via NF- $\kappa \mathrm{B}$ and $\mathrm{p} 38$ MAPK in RIE-1 cells," Biochemical and Biophysical Research Communications., vol. 378, no. 3, pp. 610-614, 2009.

[94] H. Doppler, P. Storz, J. Li, M. J. Comb, and A. Toker, "A phosphorylation state-specific antibody recognizes Hsp27, a novel substrate of protein kinase D," The Journal of Biological Chemistry, vol. 280, no. 15, pp. 15013-15019, 2005.

[95] R. A. Stetler, Y. Gao, L. Zhang et al., "Phosphorylation of HSP27 by protein kinase D is essential for mediating neuroprotection against ischemic neuronal injury," The Journal of Neuroscience, vol. 32, no. 8, pp. 2667-2682, 2012.

[96] A. Eisenberg-Lerner and A. Kimchi, "DAP kinase regulates JNK signaling by binding and activating protein kinase D under oxidative stress," Cell Death and Differentiation, vol. 14, no. 11, pp. 1908-1915, 2007.

[97] A. Eisenberg-Lerner and A. Kimchi, "PKD is a kinase of Vps34 that mediates ROS-induced autophagy downstream of DAPk," Cell Death and Differentiation, vol. 19, no. 5, pp. 788-797, 2012.

[98] R. Wani, J. Qian, L. Yin et al., "Isoform-specific regulation of Akt by PDGF-induced reactive oxygen species," Proceedings of the National Academy of Sciences of the United States of America, vol. 108, no. 26, pp. 10550-10555, 2011.

[99] L. Chen, H. Hahn, G. Wu et al., "Opposing cardioprotective actions and parallel hypertrophic effects of $\delta \mathrm{PKC}$ and $\varepsilon \mathrm{PKC}$," Proceedings of the National Academy of Sciences of the United States of America, vol. 98, no. 20, pp. 11114-11119, 2001. 


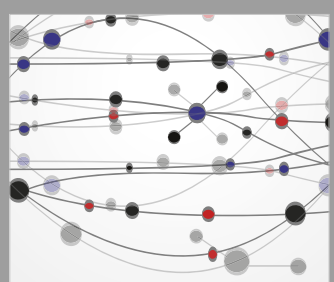

The Scientific World Journal
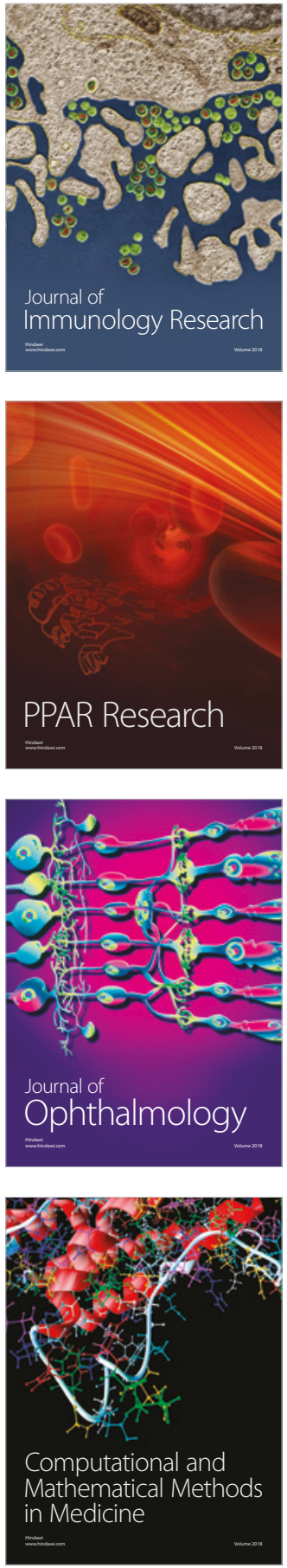

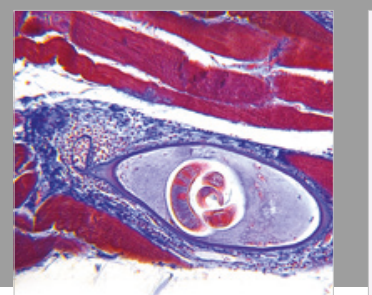

Gastroenterology Research and Practice

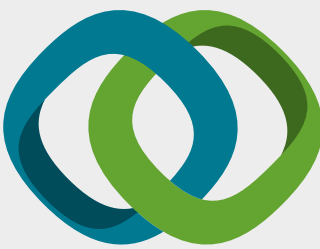

\section{Hindawi}

Submit your manuscripts at

www.hindawi.com
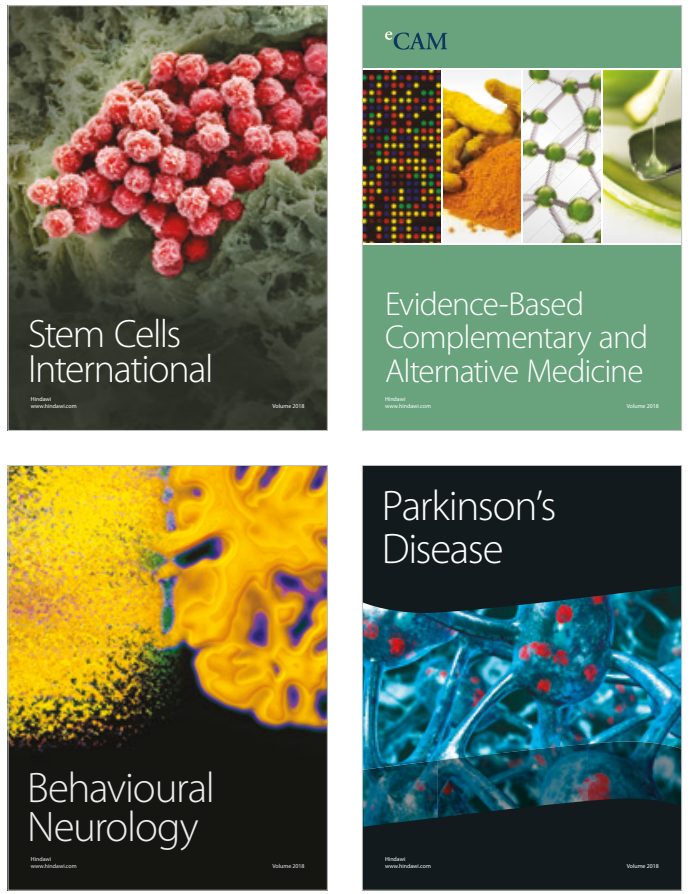

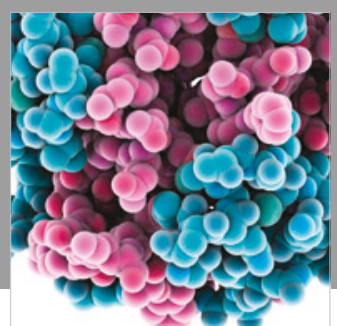

ournal of

Diabetes Research

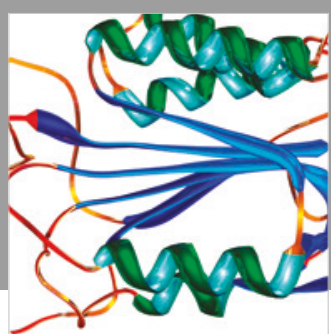

Disease Markers
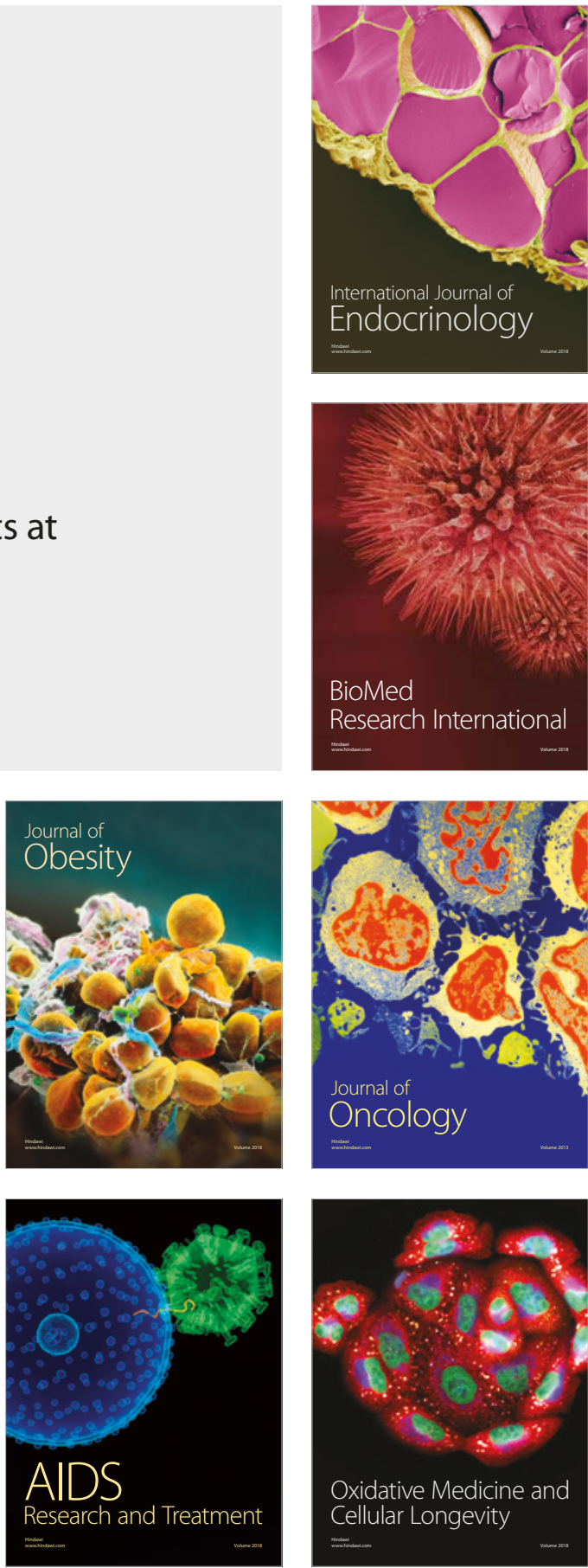